\title{
Contemplation and perception energy transition states
}

\author{
Preecha P. Yupapin ${ }^{1,2, *}$ \\ ${ }^{1}$ Asian Quantum Life Institute, 92/395 Moo 11, Supakorn Village, Sai-noi Sub-district, Sai-noi District, Nonthaburi 11150, Thailand \\ ${ }^{2}$ Interdisciplinary Research Center, Faculty of Science and Technology, Kasem Bundit University, Bangkok 10250, Thailand \\ *Corresponding author E-mail: kypreech@kmitl.ac.th
}

Copyright (1) 2015 Precha P. Yupapin (PhD). This is an open access article distributed under the Creative Commons Attribution License, which permits unrestricted use, distribution, and reproduction in any medium, provided the original work is properly cited.

\begin{abstract}
In this paper, the concept of meditation and perception energy transition states is configured described by the spirit energy transitions. Being life has been jeopardized situation due to the chaotic society. The technique that can be used to release and recover such problems is the challenge and being proposed. There are two techniques that can lead to obtain the mind concentration, where they are meditation ad mind-body considerations that have been successfully used for years. The descriptions of meditation and perception are performed by the spirit energy transitions of eight and sixteen levels respectively. The transition states and energy levels are explained, which can be useful for practitioners and world society.
\end{abstract}

Keywords: Perception; Meditation; Metaphysics; Origin of Life; Buddhism Philosophy; Quantum Life; Neuro-Quantology; Brain Science.

\section{Introduction}

After the big bang, the spirit is appeared in the forms of energies, where the origin of life is formed and confirmed by the birth and death cycle and dependent origination law by Lord Buddha [1-5]. Meditation can be used to rise up human brain performance, which has been popular practiced [6-8]. The meditation is the method that can make the spirit to be in the form of a single mind concept, which can be distinguished to be eight energy transition levels (M1 to M8. While, the Mindfulness consists of sixteen energy transition states(P1 to P16) by following the Four Mindfulness Foundation, where they are (i) Body, (ii) Sensation, (iii) Consciousness and (iv) Dhamma, where in practice, those states can be established by the mind-body consideration, in which the sequence of them is fixed without any change. Brain signal is formed by spirit within the embryo and recognized as the coherent light (photon) with a certain frequency [9-13], where the oscillation is sometimes forced by either external or internal environments. The two important states of meditation and mindfulness are the spirit oscillation time $(\Delta \mathrm{t})$ and spirit energy $\Delta \mathrm{E}$ approached zero, in which the spirit concentration and mind perception are taken placed, where finally, the meditation and perception are established. The meditation is established by single mind concept, where the reducing in nonlinear effect and noise of the brain signals are obtained [14].

The brain signals can connect and form the network throughout the body [8], thus, the change in brain signal can cause the change in being behaviors. To obtain the brain performance improvement, the technique of brain signal noise reduction has become the challenge, where there are two important techniques, medical and natural treatments. The natural treatment one has become the interesting one, where they are the meditation and mind body consideration. These two techniques are different in terms of time and energy concepts, where the meditation is concentrated on spirit oscillation time, where it is finally approached zero(stopping condition), while the mind body one is concentrated on the spirit oscillation frequency(energy), where there are four states of them, where they are (i) form, (ii) sensation, (iii) mind and (iv) mind-object [3], where the last one is the collapsed spirit state, where the spirit energy is approached zero, which is called the "nirvana" state. In this paper, the two concepts of mind concentrations are analyzed and described, where they are meditation and perception in terms of oscillation time and frequency, which can be used for brain performance improvement. The concept of brain signal is formed by the spirit oscillation and generated by two-level 
atom transition. The transition of energy is transformed to be eight and four levels by the meditative absorption and perception, respectively, which are discussed in details.

\section{Spirit energy transition model}

After big bang, spirit is a formed of energy and existed in the universe. The spirit signal is formed by the certain energy (frequency), where the embryo is active after fertilization. The spirit signal is oscillated, and the two-level atom system formed by the coherent light (photon). The oscillation frequency of the two-level atom is known as Rabi's oscillation frequency, which is defined as [10], [14]

$\mathbf{X}_{\mathrm{i}, \mathrm{j}}=\left(\mathbf{d}_{\mathrm{i}, \mathrm{j}} \cdot \mathbf{E}_{0}\right) / \hbar$

Where $\mathbf{d}_{\mathrm{i}, \mathrm{j}}$ is the transition dipole moment for the $\mathrm{i}_{\text {th }}$ to $\mathrm{j}_{\mathrm{th}}$ transition state, and $\mathbf{E}_{0=} \boldsymbol{\varepsilon} \mathrm{E}_{0}$ is the vector electric field amplitude which includes the polarization. Where $\hbar$ is the Plank's constant. The numerator has dimensions of energy, so dividing by $\hbar$ gives an angular frequency. Where $\mathbf{d}_{\mathrm{i}, \mathrm{j}}=\mathbf{d}_{\mathrm{j}, \mathrm{i}}$, it cannot assume that $\mathbf{X}_{\mathrm{ij}=} \mathbf{X}_{\mathrm{j}, \mathrm{i}}$ as $\mathbf{\varepsilon} \mathrm{E}_{0}$ may be complex, as in the case of circularly polarized light (photon. In principle, the individual being spirit frequency cannot be the same, where the being privacy is remained by the frequency distinction. The spirit energy is formed in terms of energy as $\hbar v$, which means that the active spirit is oscillated (moved) in time, alternatively, the stopped spirit, the energy is vanished.

\section{Contemplation and perception}

In the meditation, the spirit signal is confined by a single frequency (energy), where the oscillation time will be reduced and ended up by the smallest value(or zero), which means that the spirit is a single peak signal at the stopping in time situation [15]. While, in this situation the energy level is transformed from two-level atom to be eight and 16 levels with different energy values, which are shown in Figures 1 and 2, respectively. The tunable energy value is always needed to justify the energy transition state, which may be affected and caused the meditation situation, which is called an emptiness state. In this method, the certain energy value is remained, where the meditative absorption is obtained by the practical ways that they are (i) directed attention, (ii) examination, (iii) rapture, (iv)pleasure, (v) single mind, where there is no vanished energy state occurred. However, the traveling of spirit in space without time (time independence) is established, where the spirit can travel freely in time. The other method is known as mind-body consideration, we begin with $\Delta \mathrm{E} \Delta \mathrm{t} \approx \hbar$, where the spirit oscillation nonlinear effect can be reduced [3]. There are sixteen energy-level values, where they are P1 to P16 and called the perception. By using the mind-body consideration, the last remain energy is approached zero $(\Delta \mathrm{E} \rightarrow \varnothing)$. In principle, there are four situations such as (i) consciousness, (ii) sub-consciousness, (iii) unconsciousness and (iv) nirvana that can be used to describe the perception. In this situation, the spirit energy is reduced and divided to be sixteen energy levels, where the spirit the final state is oscillated without energy (no oscillation). In Figure 2, he transitions of the two-level energy system to be eight and sixteen levels are formed by meditation and mind body consideration, where they are meditative absorption and perception. The eight states of meditation absorption are originated on top and the energy and time reducing to the bottom, where in this case, the spirit oscillation time is approached zero, while the spirit energy is remained with a minimum value. The result of mind body consideration is also seen in Figure 2, where the energy is approached zero when the time goes on, in which the emptiness situation is existed within the mind-body consideration cycle, where there are sixteen energy levels, the last one is called nirvana state, where the practitioner is known as Buddhist Saint [16]. The meditation is the method that is directed to the concentration while the mind-body consideration is a cycle that the concentration is existed at the edge of the emptiness. The meditation is rarely lead to meet the nirvana sate, while the mind-body consideration is more relaxed and easier to reach the nirvana state than the meditation. However, the high level of meditation can reach the spirit stopping condition and Cerenkov radiation, in which the spirit can freely be traveling in time, aura shiny and super power holder. But the spirit energy has never been zero by the meditation; therefore, the death nirvana is difficult to obtain. In Eight Noble Paths, the perception is obtained before meditation by the following considerations as (i) body, (ii) sensation, (iii) mind and(iv) mind-object, where the sequence of them is important, where alternatively, uncertainty principle is occurred [3]. In Figure 3, the relics are formed on the noble monk (Buddha Saint), which is the everlasting material and existed in universe, which is satisfied the conservation of energy law. 

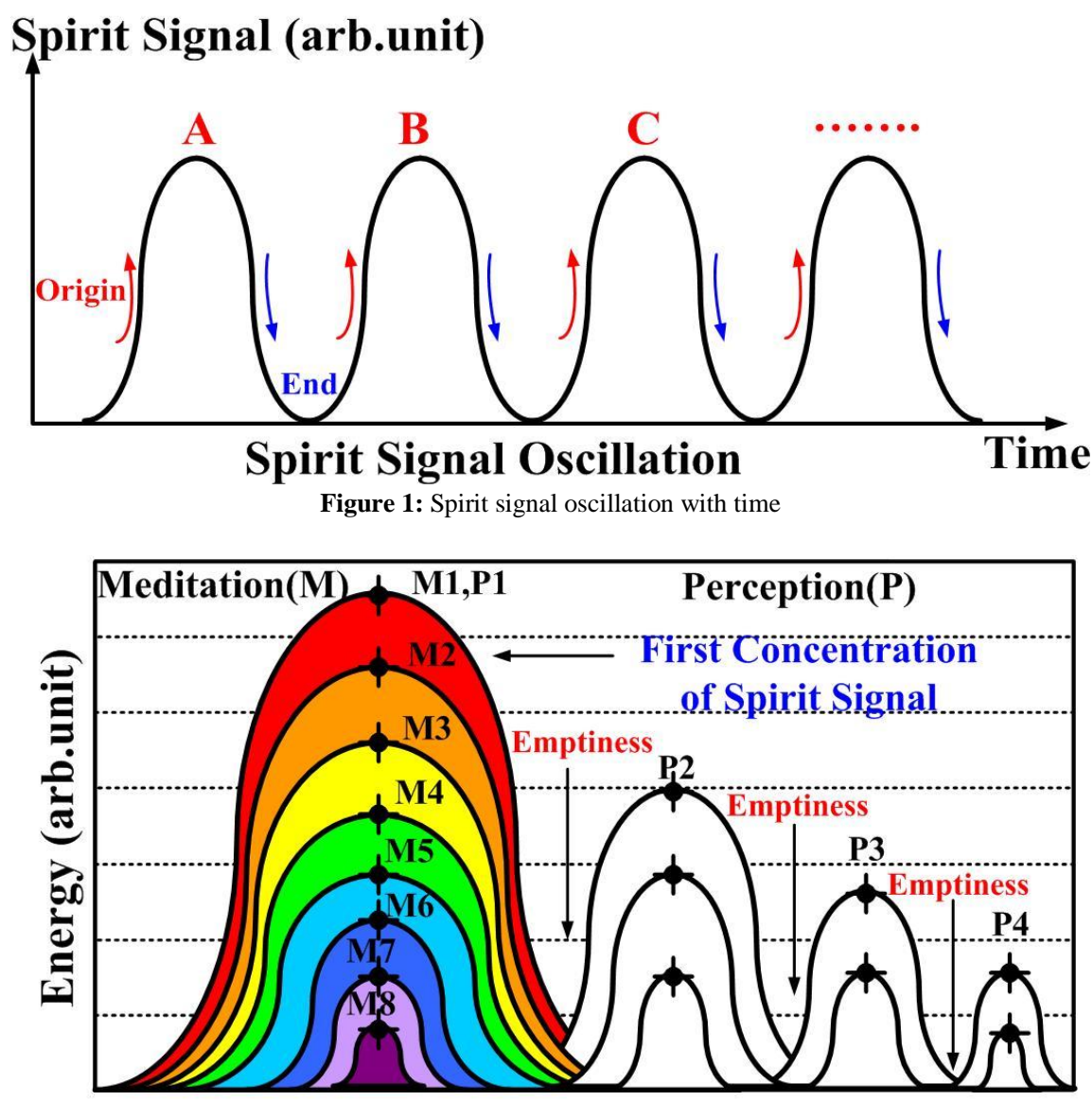

Time

Figure 2: Meditation and Perception states obtained by concentration and Mind-Object (Body) consideration. The origin and ended spirit signals are oscillated with time that can be claimed as the first digital signal concept since 2,600 years ago. There are 8 levels and 16 levels in contemplation and perception, respectively.

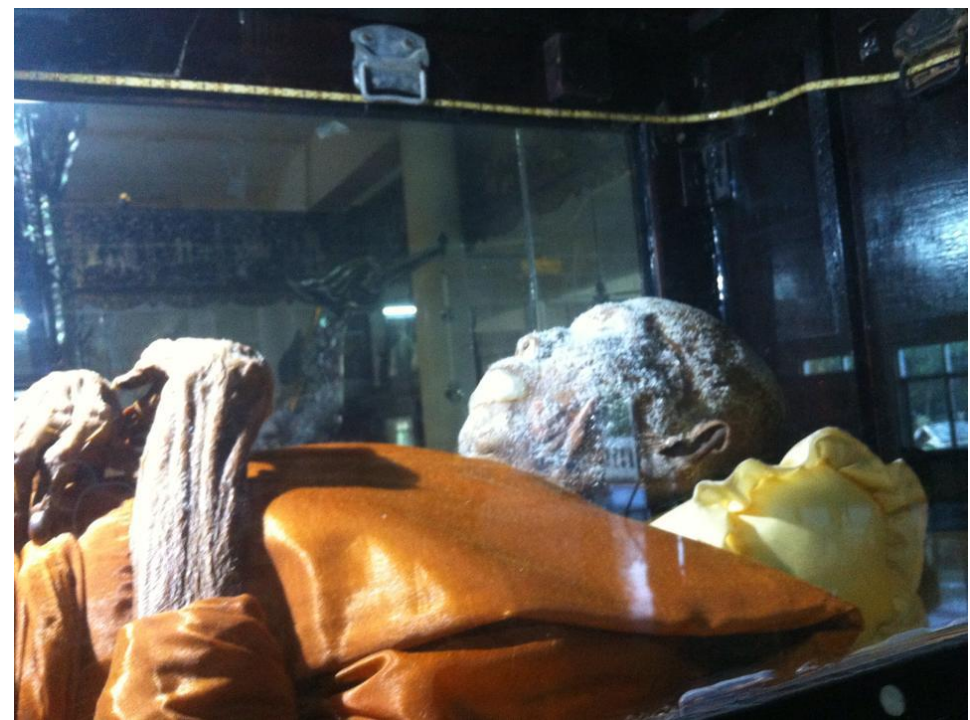

Figure 3: Noble monk body and relics at Thoong-Samakkee Dhamma Monastery, Suphanburi, Thailand in January, 2015

\section{Discussion and Conclusion}

During the deep contemplation, the speed of spirit signal oscillation in time will be decreased $(\Delta t \rightarrow \varnothing)$, from which the high energy spirit signals is approached $(\hbar v)$, where the slow speed spirit oscillation in time is occurred. This means that the conditions of high spirit energy value and slow oscillation time can lead to the black hole(nano black hole) condition, from which the spirit can travelling in time to other worlds via the warmhole. In this situation, the high gravity value 
can be used to support this concept, where the high gravity tunnel is become the fifth dimension (i.e. 3 dimensions in space, time and gravity), the gravity dimension is the new independent one from space and time.

The concepts of contemplation and perception have been described in terms of spirit signal energy distributions, in which the spirit energy is quantized and oscillated by the specific frequency. The brain signal is formed by the spirit signal after fertilization, and life has begun. The transferring of energy transition from Rabi's oscillation to eight energy levels and sixteen energy levels are explained. The concentration can be obtained by either meditation or mind-body consideration. The reductions of spirit energy and oscillation time during the perception and contemplation are also explained, which can be concluded that the nirvana state can be established by the perception, while it is rarely established by the contemplation. Alternatively, the deep contemplation may cause the risk of mind in black hole.

\section{Acknowledgment}

The author would like to give his acknowledgment to King Mongkut's Institute of Technology Ladkrabang (KMITL), Bangkok 10520, Thailand for the research laboratory facilities.

\section{References}

[1] S.W. Hawking, The Theory of Everything, 2007.

[2] P.P. Yupapin, Existence of Spirit: The Origin of Being Life, International Journal of Metaphysics, 1(1) (2014)1-4.

[3] P.P. Yupapin, Physics of Happiness and Nirvana, International Journal of Metaphysics, 1(1) (2014)1-3.

[4] S. Chotepanyo, Tripitaka, www.wattraisigkha.com, retrieved on January 5th, 2015.

[5] P.P. Yupapin, Dependent Origination Law: The Spirit Blueprint and Beyond, Life Science Journal Research, 2(3) (2015)1-4.

[6] S. Phunthawanunt and P.P. Yupapin, Science of Meditation and Therapy, Journal of Hamonized Researches in Applied Science, 2(3) (2014) 174-177.

[7] P.P. Yupapin, Birth and Death Cycle: The Scientific Approach, Life Science Journal Research, 1(3) (2014)76-79.

[8] P.P. Yupapin, Two Zero States in Buddhism: The Real Happiness, International Journal of Buddhism, 1(1) (2014)1-3.

[9] P.P. Yupapin, S. Pantian, J. Ali, Novel Design Rabi Oscillation System for Human Quantum Life Detection Probe, Life Sci J, 11(2014)235243.

[10] S. Pantian, P.P. Yupapin, THz Rabi Frequency Oscillation for Human Consciousness/sub-consciousness Detection Probe Use, J Biosensors and Bioelectronics, 4(3) (2013) e126.

[11] P.P. Yupapin S. Pantian and and J. Ali, Čerenkov Radiation: The Space-Time Paradox, Nature \& Science; 11(12) (2013)116-119.

[12] P.P. Yupapin and N. Thammawongsa, Consciousness and Sub-consciousness Detection Model under Čerenkov Radiation, Microwave \& Optical Technology Letters, 56(7) (2014) 1584-1587. http://dx.doi.org/10.1002/mop.28388.

[13] R. Jomtarak and P.P. Yupapin, Transmission Characteristics of Optical Pulse in Nested Nonlinear Microring Resonators and Gratings, Journal of Optical Society of America B, 31(3) (2014) 474-477. http://dx.doi.org/10.1364/JOSAB.31.000474.

[14] A.F.A. Noorden, K. Chaudhary, M. Bahadoran, M.S. Aziz, M. A. Jalil, C.T. Ong, J. Ali, and P.P. Yupapin, Rabi Oscillation within a Microring Resonator System, Optoelectronics Letters, 11, 2015(in press).

[15] P.P. Yupapin and N. Pornsuwancharoen, Proposed Nonlinear Microring Resonator Arrangement for Stopping and Storing Light, IEEE Photonics Technology Letters, 22(6) (2009) 404-406. http://dx.doi.org/10.1109/LPT.2009.2012503.

[16] E. William et al, Buddhist Philosophy: Essential Readings, Oxford University Press, 2009. 